\title{
TEA Laser Induced Multiphoton Dissociation of Ethylene in a Collisional Regime: Model and Experiment*
}

\author{
N. C. Peterson, ${ }^{* *}$ R. G. Manning, ${ }^{* * *}$ and W. Braun \\ Institute for Materials Research, National Bureau of Standards, Washington, D.C. 20234
}

(August 18, 1977)

\begin{abstract}
The focused beam of a pulsed $\mathrm{CO}_{2}$ TEA laser was used to photodissociate ethylene gas at total pressures of 100-300 torr. The laser pulse induces molecular hydrogen elimination to vield primarily $\mathrm{C}_{2} \mathrm{H}_{2}$ and $\mathrm{H}_{2}$ in an large excess of helium to eliminate heating. An invesigation was made of the dependence of product yield on wavelength, inert gas pressure, ethylene pressure and intensity. Photochemical yield was followed over a 10,000 fold range. Its dependence was investigated employing alternate methods of focusing the laser beam, one of which results in a constant volume geometry. A photometric method of characterizing this focal zone is described.

A "structureless" computer model of the laser pumping process involving coupled rate equations and phenomenological absorption and stimulated emission coefficients is described. The model assumes rapid intramolecular energy transfer between the various vibrational modes throughout the entire vibrational manifold, and rapid equilibration of rotational states. The model includes stimulated emission and deactivating collisions, and predicts a product yield versus intensity dependence that does not exhibit threshold behavior in agreement with experiment. Calculated dependences of product yield on laser power for various parameters are given in order to relate the model to various photolysis experiments.
\end{abstract}

Key words: Infrared: kinetics; lasers; model; multiphoton; photochemistry.

\section{Introduction}

Since it was first shown that molecules can be dissociated by multiphoton absorption of high intensity $\mathrm{CO}_{2}$ laser radiation [1], ${ }^{1}$ this process has been shown to be molecular specific by demonstration of isotopic selectivity [2, 3]. There have followed a considered number of papers discussing mechanisms of the process and advocating reasonable physical models [4-15]. To test any model, detailed experimental studies are required to determine the influence of parameters such as substrate pressure, total pressure, laser wavelength and laser intensity on the photodissociation process. The present investigation was primarily concerned with the photofragment yield and its dependence on the incident laser intensity. A knowledge of the overall chemistry of the system is required leading to both primary and secondary products. In addition, a knowledge of the photolysis zone geometry and the intensity distribution in the laser beam is required.

The present paper reports results on the photodissociation of $\mathrm{C}_{2} \mathrm{H}_{4}$ in a high intensity, focused $\mathrm{CO}_{2}$ laser beam in which the photolysis involves direct dissociation into $\mathrm{C}_{2} \mathrm{H}_{2}$ and $\mathrm{H}_{2}$ via molecular hydrogen elimination. This is the "thermal" channel for the dissociation of the $\mathrm{C}_{2} \mathrm{H}_{4}$ molecule which has the lowest activation energy. The ethylene photolysis follows the general pattern for dissociation of molecules which was outlined in a previous study [16]. There are no secondary rections occurring in this system, so that measurement of the $\mathrm{C}_{2} \mathrm{H}_{2}$ yield provides a direct measure of the dissociation of

* Contents of this paper presented at the Physics of Quantum Electronics 2nd Winter Colloquin on Laser Induced Chemistry, Park City, UT, Feb. 13-16, 1977. This paper was supported in part by the Energy Research and Development Administration under contract number EA-77-A-01-6010.

** Guest worker, Laser Chemistry Program, Institute for Materials Research, Summer, 1976 nanent address: Department of Chemistry, Polytechnic Institute of New York, 11201

*** NRC-NBS Postdoctoral Associate 1975-1977. Permanent address: SRI International, Menlo Park, CA 94025.

Figures in brackets indicate the literature references at the end of this paper.
$\mathrm{C}_{2} \mathrm{H}_{4}$. The photodissociation yield was measured as a function of several parameters, in particular, the laser intensity. Two focusing configurations were used in these experiments permitting us to characterize the magnitude of the photolysis volume. We have developed a kinetic model describing the photolysis mechanism as a stepwise pumping process and present the results of calculations of product yield as a function of laser intensity. The experimental results are then compared with the prediction of the model. The model serves as a guide to predict the sensitivity of various experimental and molecular parameters, details of the pumping and collisional deactivation processes.

\section{Experimental Procedure}

The general experimental arrangement was described previously $[16,17]$. A $\mathrm{CO}_{2}$ TEA laser beam, $(\sim 0.5$ joule $)$ is either focused directly into a $1 \mathrm{~cm}^{3}$ sample photolysis cell using a $10 \mathrm{~cm}$ focal length $\mathrm{NaCl}$ lens, or is directed at an acute angle toward a $30 \mathrm{~cm}$ focal length spherical mirror and the emerging reflected beam is then focused through a $10 \mathrm{~cm}$ focal length lens into the photolysis cell. In the first focusing configuration the photolysis volume is conical, in the second, the image in the cell is astigmatic. The sample cell is connected through a two position, six port gas chromatograph sampling valve to either a gas handling system, or to a flame ionization gas chromatograph.

After one or more laser pulses, the sample, usually consisting of about $50-200$ torr $(1$ torr $=133.3 \mathrm{~Pa}$ ) total pressure of a 1 percent solution of $\mathrm{C}_{2} \mathrm{H}_{4}$ in helium, was admitted into the chromatograph column and the dissociation product, $\mathrm{C}_{2} \mathrm{H}_{2}$, analysed. Total sample decomposition was usually maintained at about 5 percent or less by varying the number of laser pulses. The laser pulse rate was $1 \mathrm{~Hz}$. The 
sensitivity of the instrument toward $\mathrm{C}_{2} \mathrm{H}_{4}$ and $\mathrm{C}_{2} \mathrm{H}_{2}$ was determined such that accurate percentage decompositions could be measured. Mixtures were prepared using calibrated Bourdon gauges. Laser power measurements were made using a thermopile detector. The laser wavelength was monitored using a spectrum analyser. Laser intensity was varied by using calibrated wire screens as attenuators. These screens were calibrated with respect to total transmittance, determined using a visible radiation spectrophotometer. The screens were then calibrated with respect to transmittance in the zero and higher diffraction orders [18-20]. This was done by using $\mathrm{cw} \mathrm{CO}_{2}$ laser radiation in Fraunhofer diffraction geometry: A screen was placed in front of a convex lens and a detector with a $1 \mathrm{~mm}^{2}$ active sensor area was mounted on a translation device and positioned at the focus. The intensity of the zero order diffraction maximum could be measured and the first order intensity maximum could be measured by translating the detector.

After focusing, the intensity in higher diffraction orders at the respective focal spots is considerably less than the intensity in the zero order focal spot, thus only radiation in the zero order diffraction maximum results in appreciable photolysis in the reaction cell. To test this hypothesis and to demonstrate that there is no overlap between the zero order maximum and higher diffraction orders in the focal region, a number of screens with the same total transmittance but different zero order transmittance were used to attenuate the beam. Product yields always followed the zero order transmittance; screens with the highest zero order intensity always yielded the highest product yields.

An attempt to characterize the focal spot size using combinations of screens was also made. The separation of the zero and first order focal spots is given by the divergence multiplied by the focal length of the lens. The divergence is $\lambda / d$, where $\lambda$ is the laser wavelength and $d$ is the distance between the wires in the screen. It is, in principle, possible to increase the screen spacing to a point where first order and zero order spots overlap. When this condition is achieved, product yields should follow the total screen transmittance rather than the zero order transmittance. It was not practical to achieve this condition because spacings between the wires would have had to have been too wide to accommodate a sufficient number of wires in the laser beam in the present experimental arrangement.

Photometry was performed in order to evaluate the intensity distribution in the laser beam, the image size at the focus and the depth of the focal region of the astigmatic image. It was found that exposed and developed photographic film could be reproducibly bleached by the focused laser beam. If the intensity of the beam is varied by means of calibrated screens a wide range of intensity can be recorded on film covering the range from saturation (complete bleaching) to a faint, barely discernible image. The useful range of laser intensity over which changes in intensity can be clearly observed is somewhat more than one order of magnitude. This range can be extended by varying the density of the exposed film. All intensity estimates were made visually by comparing the intensity of an image against a set of images of known relative intensity obtained through the use of calibrated screens. Screens do not change the image size at the focus but do produce resolved multiple images from the higher order diffraction maxima at high laser intensities. Figure 1 displays images of the laser beam at the focus of a $30 \mathrm{~cm}$ focal length mirror. To obtain these images, the laser

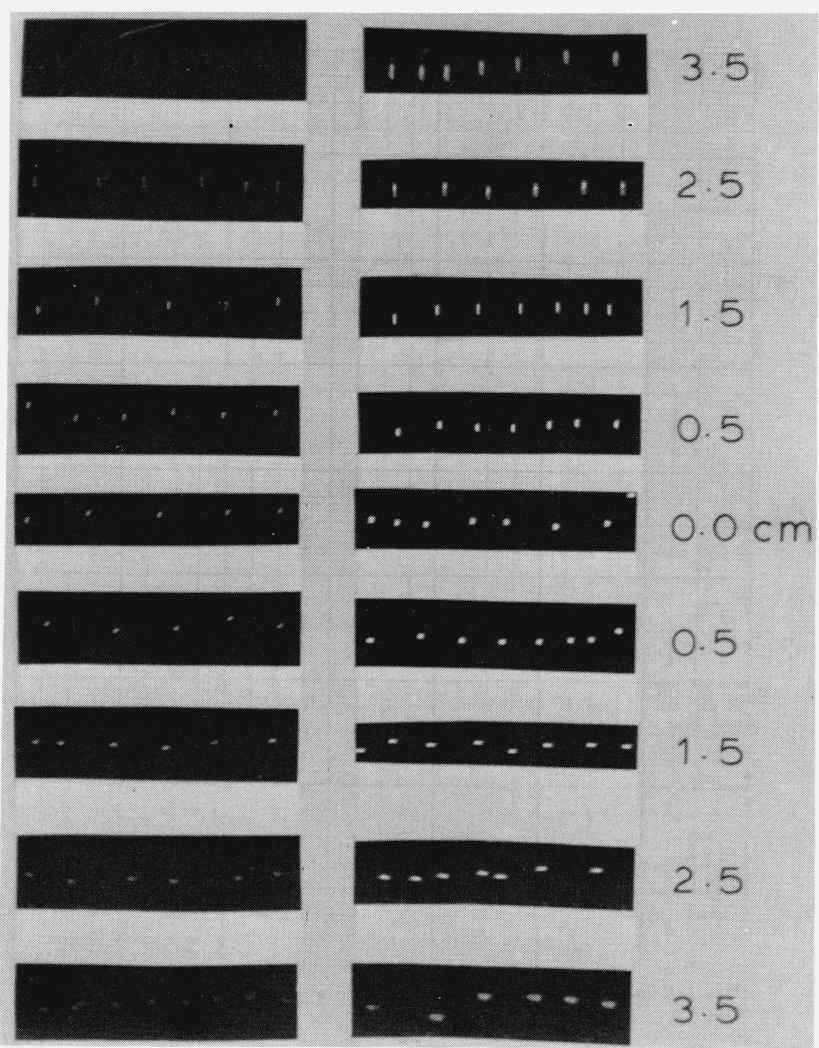

FIGURE 1. Multiple bleached images on exposed and developed film produced by a focused laser in the vicinity of the focus $(30 \mathrm{~cm}$ focal length spherical mirror in astigmatic configuration); Left vertical sequence of shots at lower laser intensity than right sequence.

From top to bottom different focusing positions roughly centered about the circle of least confusion, labeled $0.0 \mathrm{~cm}$.

beam was incident to the mirror at an oblique angle. The reflected focused image can be seen from figure 1 to be highly astigmatic. The position labeled $0.0 \mathrm{~cm}$ is approximately at the circle of least confusion. This position is about midway between the principal and secondary focal ellipses which are about $1.5 \mathrm{~cm}$ from the nearly circular image position. From calibrations of film bleaching versus known relative laser intensity in several series of exposures, it can be seen that the laser intensity is approximately constant within $1.5 \mathrm{~cm}$ of the central circular image and decreases by a factor of over two between 1.5 and $2.5 \mathrm{~cm}$.

By measuring the size of the bleached image on the film using a magnifier it is seen that below some laser intensity the image size is independent of the laser intensity. Although figure 1 appears to show that the weaker exposure of the central image (on the left) is smaller than the brighter central image (on the right), this is an optical illusion. By observing the two images under a measuring microscope it is seen that the two central images are equal.

The measured focal volume of $30 \times \pi(1.2 / 2)^{2}=34 \mathrm{~mm}^{3}$ obtained from the $3.0 \mathrm{~cm}$ range of focus and $1.2 \mathrm{~mm}$ diameter (measured diameter of the circular image) can be used to calculate the focal volume in the photolysis cell which results from focusing the converging beam from the spherial mirror with a concave $10 \mathrm{~cm}$ focal length lens. The 
measured focal length of the mirror and lens combined is 6 $\mathrm{cm}$. The $34 \mathrm{~mm}^{3}$ focal volume measured with the spherical mirror alone is therefore reduced by a factor of $(30 / 6)^{3}$, i.e., by the cube of the ratio of the focal length of the mirror to the focal length of the combined optic. This results in a focal volume in the photolysis cell of $\sim 0.27 \mathrm{~mm}^{3}$ and an effective cross-section of $\sim 0.045 \mathrm{~mm}^{2} \cong 5 \times 10^{-4} \mathrm{~cm}^{2}$ for the astigmatic images obtained in these experiments. This measured cross-section can then be used to determine the laser energy fluence. The maximum laser energy used in these experiments was about 0.5 joule per pulse, so that the energy fluence is about $0.5 / 5 \times 10^{-4} \sim 1000$ joules $/ \mathrm{cm}^{2}$. As a point of reference this energy fluence is about two orders of magnitude larger than required to disociate $\mathrm{SF}_{6}$ as recently reported by Ambartzumian et al. [9] and by Campbell, Hancock, and Welge [21]. The photon fluence at $1040 \mathrm{~cm}^{-1}$ equivalent to an energy fluence of $10^{3}$ joules $/ \mathrm{cm}^{2}$ is $5 \times 10^{22}$ photons $/ \mathrm{cm}^{2}$ in each pulse.

Figure 2 displays the relative product yield per laser pulse as a function of relative laser intensity in two distinctly different focusing configurations. In the first, the TEA laser is focused directly by impinging the laser beam perpendicular to a $10 \mathrm{~cm}$ focal length $\mathrm{NaCl}$ lens which focuses the laser at the center of the photolysis cell. This "tight" focusing results in a product versus (intensity) ${ }^{3 / 2}$ dependence which is well known to be purely a consequence of conical focusing geometry and has been discussed recently [22]. Because of the changing photolysis volume this "perfect" focusing gives no information about the "true" dependence of photolysis yield on the laser intensity.

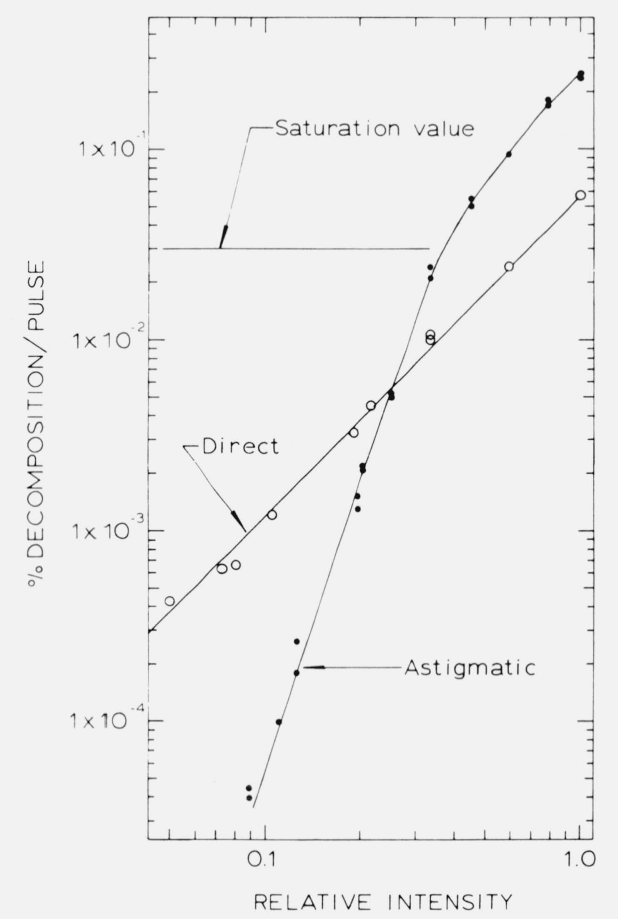

Figure 2. Dependence of percent decomposition of $\mathrm{C}_{2} \mathrm{H}_{4}$ per laser pulse on the laser intensity for direct focusing, and for astigmatic geometry.

Experimental conditions: $\mathrm{P}_{\mathrm{C}_{2} \mathrm{H}_{4}}=0.625$ torr, $\mathrm{P}_{\mathrm{He}}=200$ torr, irradiation using $\mathrm{P} 14,(10.6 \mu)$
The situation is quite different if astigmatic focusing is employed (see fig. 2). At low intensities the product yield is a strongly increasing function of the laser intensity in a zone of approximately uniform intensity while at high laser intensity all of the reactant molecules in this zone are converted to products. The approximate saturation level is shown in figure 2. At intensities greater than the saturation intensity the photolysis volume extends to either side of the central constant intensity zone. This extended volume is conical and the observed product dependence on intensity closely follows the $I^{3 / 2}$ dependence.

The "saturation volume" can be calculated from the measured percent decomposition/pulse and the total photolysis cell volumle, $V_{T}=1000 \mathrm{~mm}^{3}$ from the following expression: $(\%$ decomposition/pulse $) \times(1 / 100) \times V_{T}=0.30$ $\mathrm{mm}^{3}$. This value is in excellent agreement with the value of $0.27 \mathrm{~mm}^{3}$ obtained from the size of the photolysis volume obtained from the photometric measurements. Astigmatic focusing in the region below the saturation intensity leads to a "correct" dependence of product yield on laser intensity provided that the laser intensity is uniform in the beam, i.e., that there are no "hot" spots in the beam.

A third focusing mode was attempted which was similar to that employed by Campbell et al. [21]. A long focal length lens was used to focus the laser beam down to a small diameter with approximately uniform intensity throughout the length of photolysis cell. It was not possible to achieve an intensity high enough to effect detectable photolysis of ethylene in this focusing mode. This fact is not surprising in view of the present observation than an intensity two orders of magnitude higher is required to dissociate appreciable amounts of $\mathrm{C}_{2} \mathrm{H}_{4}$ compared to that required to photodissociate $\mathrm{SF}_{6}$.

\section{Results and Discussion}

\subsection{Mechanism of $\mathrm{C}_{2} \mathrm{H}_{4}$ Photolysis}

The photolysis of ethylene yields primarily acetylene and molecular $\mathrm{H}_{2}$ by the process (1):

$$
\mathrm{C}_{2} \mathrm{H}_{4} \stackrel{n h \nu}{-} \mathrm{C}_{2} \mathrm{H}_{4} * \rightarrow \mathrm{C}_{2} \mathrm{H}_{2}+\mathrm{H}_{2} \quad n \geq 26
$$

There is no significant $\mathrm{H}$ atom elimination by process (2).

$$
\mathrm{C}_{2} \mathrm{H}_{4} \rightarrow \mathrm{C}_{2} \mathrm{H}_{3}+\mathrm{H}
$$

Products such as $\mathrm{C}_{2} \mathrm{H}_{6}, \mathrm{CH}_{4}, \mathrm{C}_{3} \mathrm{H}_{6}$, or $\mathrm{C}_{4} \mathrm{H}_{10}$ are expected to result through processes (3) and (4) followed by various radical and $\mathrm{H}$ atom combination steps. These products were not detected.

$$
\begin{aligned}
& \mathrm{C}_{2} \mathrm{H}_{4}+\mathrm{H}+\mathrm{M} \rightleftarrows \mathrm{C}_{2} \mathrm{H}_{5}{ }^{*}+\mathrm{M} \rightarrow \mathrm{C}_{2} \mathrm{H}_{5}+\mathrm{M} \\
& \mathrm{M}+\mathrm{C}_{2} \mathrm{H}_{5}+\mathrm{H} \rightarrow \mathrm{C}_{2} \mathrm{H}_{6}{ }^{*}+\mathrm{M} \rightleftarrows \mathrm{C}_{2} \mathrm{H}_{6}+\mathrm{M} \\
& \mathrm{C}_{2} \mathrm{H}_{6}{ }^{*} \quad \rightarrow 2 \mathrm{CH}_{3}
\end{aligned}
$$

Excited ethylene $\mathrm{C}_{2} \mathrm{H}_{4}{ }^{*}$, similar to that produced by process (1), can also be prepared chemically through reaction (chemical activation) $[23,24]$, as in process (5), or in a high temperature environment such as that produced in a shock tube. Ethylene, produced by chemical activation is known to 
dissociate by a molecular hydrogen elimination process. Dissociation via process (1) therefore represents the channel having the lowest activation energy for thermal dissociation of $\mathrm{C}_{2} \mathrm{H}_{4}$.

$$
\mathrm{CH}_{2}(\mathrm{~T})+\mathrm{CH}_{2}(\mathrm{~T}) \rightarrow \mathrm{C}_{2} \mathrm{H}_{4} * \rightarrow \mathrm{C}_{2} \mathrm{H}_{2}+\mathrm{H}_{2}
$$

Evidence in support of product formation by photodissociation rather than through a laser heating is primarily the following: photolysis of a mixture of ethylene and a second substance when photolysed by the same i.r. laser pulse yields a product distribution which is the sum of products obtained from each substance photolysed separately. This diagnostic has been applied previously [16] and is also discussed in more detail in a recent report [25].

\subsection{Characterization of Photolysis Volume}

It is important to characterize the photolysis zone in order to allow determination of the photon fluence and the saturation volume, the volume in which complete dissociation occurs. These parameters are necessary to compare experimental results with any theoretical predictions. As indicated above, our approach to this problem was to use a photometric calibration scheme.

The results of the experiments lead to the following conclusions.

(1) Direct focusing leads to an experimental product yield dependence proportional to the 1.5 power of the laser intensity. This corresponds to complete dissociation of the ethylene in some portion of the focal cone, the volume of which increases in a manner predictable by geometrical considerations.

(2) Astigmatic focusing leads to a photolysis zone of essentially constant volume, and at high intensity, appreciable photolysis in the conical region outside of this zone.

(3) The volume of the photolysis zone obtained by photometry is $0.27 \mathrm{~mm}^{3}$. The transition from the $3 / 2$ power law for photolysis yield provides an independent estimate of the uniform photolysis volume of $0.30 \mathrm{~mm}^{3}$.

(4) The dependence of product yield versus laser intensity as the intensity is reduced below the saturation level characterizes the effect of intensity on yield in a constant volume region. The photometric experiments do however reveal variations of intensity in the astigmatic focal region. The effect of intensity variation is discussed below.

(5) There is no evidence for threshold behavior in these experiments. The detection sensitivity limits the amount of photolytic product observed at low laser fluence.

\section{Kinetic Model for Photolysis}

A kinetic model for the laser pumping photolysis was developed which provides qualitative agreement with the dependence of photolysis yield upon laser intensity in the uniform photolysis zone. This model has been useful in planning further experimental work.

The physical basis for the model is incorporated in the following assumptions:

(1) At the inert gas pressures used in these experiments, the rotational states are equilibrated by collisional energy transfer $(R-T)$ in times short compared to the laser pulse.
(2) The vibrational states of the molecule are in rapid communication with each other, i.e., there is fast intramolecular vibrational energy transfer, in times much shorter than the laser pulse. Unimolecular rate studies require this idea.

(3) The density of states accessable to laser pumping increases with energy proportional to $(n h \nu)^{s-1}$. This is an approximation formula which has been used in unimolecular rate theory [26].

(4) There are no exact coincidences of the laser emission frequency and the molecular absorption line. Some small adsorption probability is associated with the pressure broadened tail of a rotational-vibrational absorption profile.

(5) Absorption cross-sections have not been measured for most of the spectroscopic transitions required for the multiphoton process. We therefore have taken them to be equal. The predictions of this model are relatively insensitive to absorption cross-section. Changing absorption cross-sections can be incorporated in a phenomenological way as required to best fit the experimental data.

(6) The absorption process is treated by coupled rate equations for the time dependent population of all of the excited states, i.e., the optical pumping is a stepwise process.

(7) Highly excited molecules lose energy via $V-T$ energy transfer collisions. We have assumed that the probability of an energy loss process is proportional to the density of states at the lower energy. $V-V$ (intermolecular) processes are not included, therefore this model describes experiments where $V \rightarrow R, T$ processes dominate over $V \rightarrow V$. This is probably a good approximation if low pressures of substrate are photolysed in the presence of high pressures of inert gas.

(8) The $N$ th stage in the absorption process is taken as irreversible and leads to dissociation. The product yield is determined by the population in the $N$ th energy state after the laser is turned off. Dissociation terminates at the end of the laser pulse. This is consistent with assumption (7) above.

The set of coupled equations, considering only opticalpumping and stimulated emission is

$$
\begin{aligned}
d Z_{i} / d t & =-\phi(t) \epsilon_{1}\left[Z_{1}-B_{2} Z_{2}\right] \\
& \cdot \\
& \cdot \\
& \cdot \\
d Z_{i} / d t & =\phi(t) \epsilon_{i-1}\left[Z_{i-1}-B_{i} Z_{i}\right]-\phi(t) \epsilon_{i}\left[Z_{i}\right. \\
& \left.\cdot \quad-B_{i+1} Z_{i-1}\right], 1<i<N \\
& \cdot \\
& \cdot \\
d Z_{N} / d t & =\phi(t) \epsilon_{N-1} Z_{N-1}
\end{aligned}
$$

where $Z_{i}$ is the concentration of molecules in all states at the $i$ th energy level, $\phi(t)$ is the light intensity, photons $\mathrm{s}^{-1} \mathrm{~cm}^{2}$, $g_{i}$ is the $i$ th level degeneracy, i.e., the number of states in the vicinity of the $i$ th energy level approximated by $g_{i}=$ $(i)^{s-1}$, where $s$ is the number of vibrational degrees of freedom in the molecule. The quantity $B_{i}$ is the ratio of the degeneracy of the $i-1$ level to that of the $i$ th level. The quantity $\epsilon_{i}$ is the average phenomenological absorption cross-section for absorption from the $i$ th to the $(i+1)$ level and is the sum of all the possible absorption cross-sections from each sublevel of the $i$ th energy index to each accessible sublevel of the $(i$ +1 ) index divided by the degeneracy of the $i$ th level. The cross-section for stimulated emission is similarly defined as the sum of cross-sections from each sublevel of the $(i+1)$ 
index to each possible sublevel of the $i$ th level divided by the degeneracy of the $(i+1)$ level. As a consequence of the definition of $\epsilon$ 's

$$
\epsilon \uparrow=\epsilon \downarrow \frac{\mathrm{g}_{i+1}}{\mathrm{~g}_{i}} \text { or } \epsilon \uparrow \mathrm{B}=\epsilon \downarrow
$$

where $\epsilon \uparrow$ is the cross-section for absorption and $\epsilon \downarrow$ is the cross-section for stimulated emission [27].

In order to determine the overall efficiency of the pumping process with respect to the intensity of the laser beam, numerical integration was carried out using the set of coupled differential equations (I) taking $Z_{i}=0$ at $t=0$ for $i>1$.

The time dependence of the laser pulse intensity was taken as parabolic [28] and is given by equation (III).

$$
\begin{aligned}
\phi(t)= & \phi_{\max }(t / \tau)[2-(t / \tau)] \\
& \text { photons } \mathrm{ns}^{-1} \mathrm{~cm}^{-2}, 0 \leq \mathrm{t} \leq 2 \tau \\
\phi(t)= & 0, t>2 \tau
\end{aligned}
$$

In the absence of any deactivation steps the time dependent pulse shape does not affect the pumping process. The calculations were performed taking the initial reactant concentration as $10^{13}$ molecules $/ \mathrm{mm}^{3}$. The total number of pumping levels is a parameter, equivalent to the energy required to dissociate the molecule, i.e., the population reaching the $N$ th level is counted as product. The calculation involves multistep excitation and stimulated emission up to the $(N-1)$ th level, dissociation of activated molecules pumped into the $N$ th level is assumed irreversible, i.e., there is no back flux from the $N$ th (product) level to the $(N-1)$ level.

Figure 3 displays calculations of product yield (molecules $\mathrm{mm}^{-3}$ ) versus $\phi_{m} \cdot \epsilon$ taking the number of levels required to dissociate the molecule as a parameter. For these calculations the density of states at each energy was taken to be unity, i.e., the molecular manifold of states is similar to that of a diatomic molecule. The model calculation gives an efficiency for photodissociation which is strongly dependent on the number of levels required to dissociate the molecule. The value $N=26$ is approximately the number of photons required to bring the ethylene molecule to its activation energy for dissociation (which is $\sim 73 \mathrm{kcal} / \mathrm{mol}$ [29]). For a $N=26$ level system, the dissociation efficiency is almost two orders lower than for a $N=6$ level system. The slope of product yield versus $\phi_{m} \cdot \epsilon$ (intensity) in the limit of low intensity approaches $(N-1)$ for $N=6$ and $N=12$ but is not reached for the $N=26$ level system in the range of the calculation. This can be seen in figure 4 .

The effect of the parameter $s$, which defines the density of states at each energy index $i$, on the absorption as well as stimulated emission process is shown in figure 5 . As s, the number of oscillators increases, for a fixed value of $N$ (26 in this case) the photolysis efficiency increases, and also the slope of the yield versus intensity dependence increases, i.e., the approach towards saturation is considerably steeper [30].

Collisional deactivation $(V-T)$ steps are included into the model to investigate the effect of collisions on the shape of the product versus intensity curve. Two additional terms are added to the rate expression equations (I) as shown in (IV).

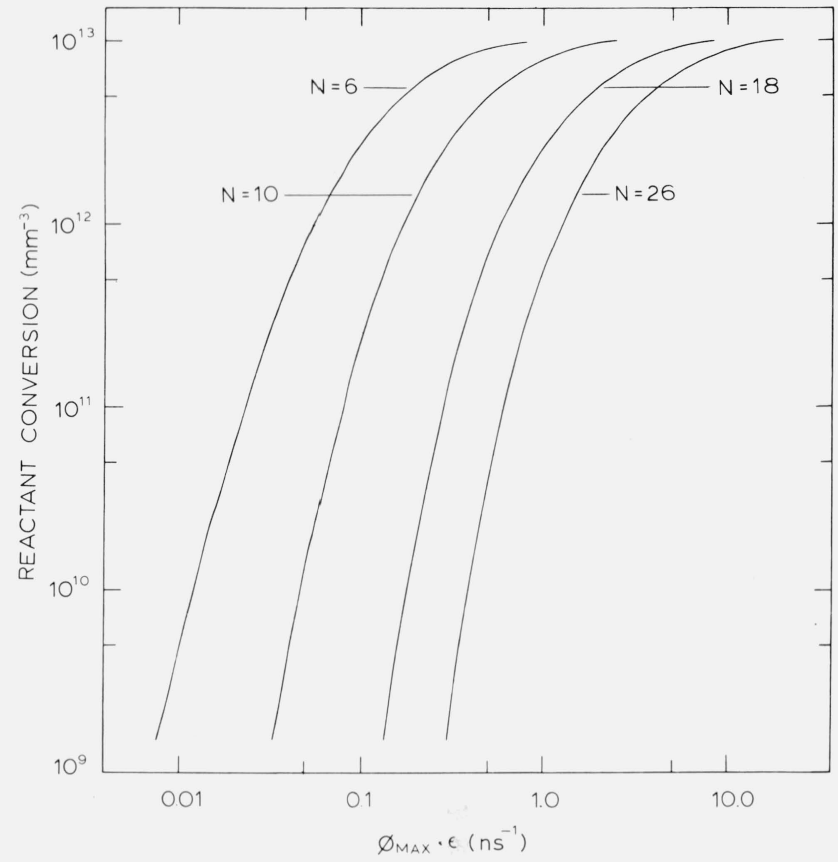

Figure 3. Curves: Reactant conversion to product versus $\phi_{\max } \boldsymbol{\epsilon}$ as a function of the number of steps required to photodissociate a molecule; no deactivation, parameter $\mathrm{s}$ is 1 .

Curves saturate at $10^{13}$ molc $\mathrm{mm}^{-3}$, the initial concentration of reactant. The absorption coefficient $\epsilon_{1}$ is the same for all levels.

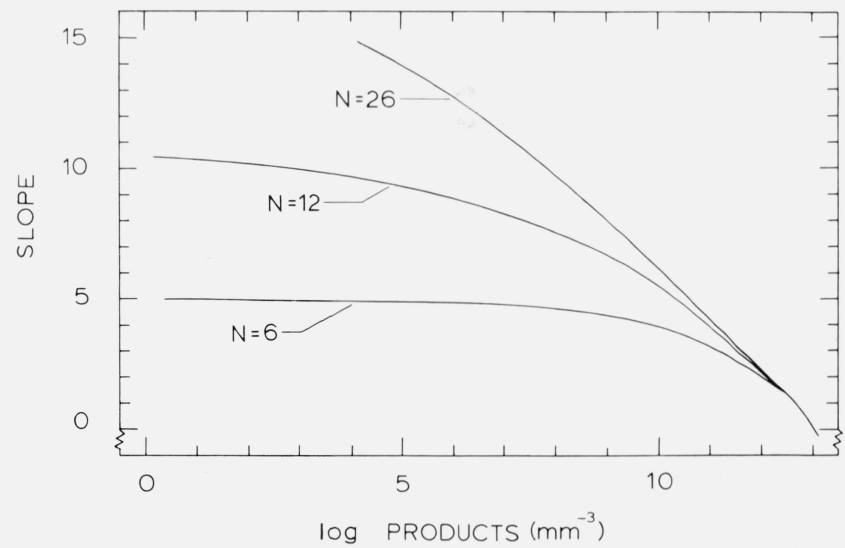

Figure 4. Dependence of the slope of the yield-intensity curve in Figure 3 on the product yield $\left(\operatorname{molc} \cdot \mathrm{mm}^{-3}\right)$.

$$
\begin{aligned}
d Z_{i} / d t=\frac{\text { pumping }}{\text { processes }} & +D \sum_{k=i+1}^{N-1} \frac{g_{i}}{g_{k}} Z_{k} \\
& -D \sum_{k=1}^{i-1} \frac{g_{k}}{g_{i}} Z_{i}
\end{aligned}
$$




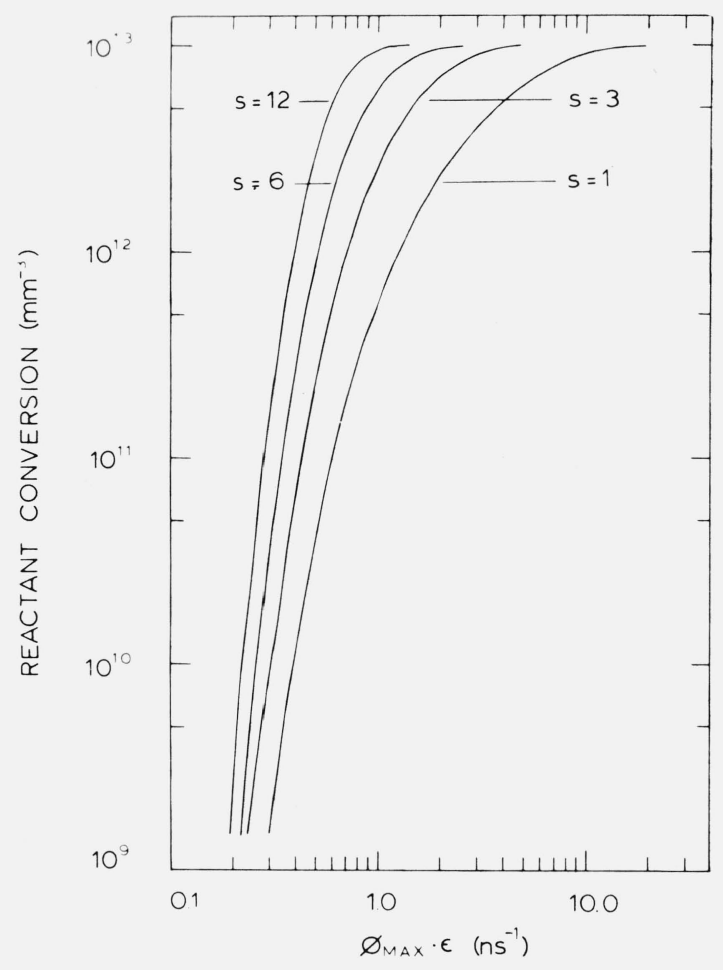

Figure 5. Plot of reactant to product conversion $\left(\mathrm{molc} \cdot \mathrm{mm}^{-3}\right)$ versus $\phi_{\max } \cdot \boldsymbol{\epsilon}(n s)^{-1}$ versus $\mathrm{s}$, the number of effect vibrational degres of freedom in the molecule, with $\mathrm{N}=26$, no deactivation and each $\epsilon_{i}=$ constant.

Deactivation is assumed to occur from any level $k<N$ to any lower level $i$, with smaller energy loss steps more probable than larger steps. The energy loss steps are assumed proportional to the density of states, encompassed in the ratios of $g$ 's in eq (IV). The parameter $D$ is in similar units to the rate parameter $\phi(t) \cdot \epsilon$ and can be simply related to the collision frequency. Units of $D$, consistent with the units of $\phi(t) \cdot \epsilon$, are (nanoseconds) $)^{-1}$ and $D\left(10^{+9}\right) \mathrm{s}^{-1}=k_{b i}$ (deactivation) $\cdot(M)$, where $k_{b i}$ (deactivation) is the bimolecular deactivation rate constant and $(M)$ is the concentration of deactivator. For helium as the deactivator $k_{b i} \approx 4 \times 10^{-10} \mathrm{~cm}^{3} \mathrm{molc}^{-1} \mathrm{~s}^{-1}$. Thus a pressure of about 8 torr helium is equivalent to a value of $D \approx 0.1$ (nanoseconds) ${ }^{-1}$, assuming a deactivation step for every collision.

Figure 6 shows the effect of deactivation on the multiphoton dissociation process. As $D$ increases above 0.01 (negligible deactivation) the conversion to product becomes less efficient. The slopes of yield versus intensity are not significantly affected at low product conversion but approach saturation more abruptly than does the zero deactivation curve.

In the absence of any experimental evidence for the size of the $\boldsymbol{\epsilon}_{i}{ }^{\prime}$ s we have assumed that $\boldsymbol{\epsilon}_{i}$ varies slowly with $i$. In these calculations the absorption coefficients for each energy level are taken to be the same. The magnitude of the $\epsilon$ 's required to similate dissociation comparable to experiment are physically reasonable [31].

The kinetic model can be related to a picture of the pumping process like Bloembergen's which concentrates on the (inefficient) spectroscopic transitions followed by easy pumping stages in a quasi-continuum. In the kinetic model for $N=26$ the $\epsilon_{i}$ 's were divided into two groups, group $\mathrm{L}$ for $i=1-5$ and group $\mathrm{H}$ for $i=6-26$. Calculations were performed with ratios of the $\epsilon_{i}$ 's for the $H$ group to those of the $\mathrm{L}$ group of 1,10 , and 100 . The results of these calculations are shown on figure 7 by curves D, C, and B respectively. Curve A refers to the model prediction for $N=$ 6 and with the same $\epsilon$ 's as for curve D. These curves show that at low conversion the yield is predicted to increase for curve $B$ relative to $C$ and $D$, but the slopes of yield-intensity for $\mathrm{B}, \mathrm{C}$, and D are remarkably similar and steeper than for the $N=6$ result (curve A).

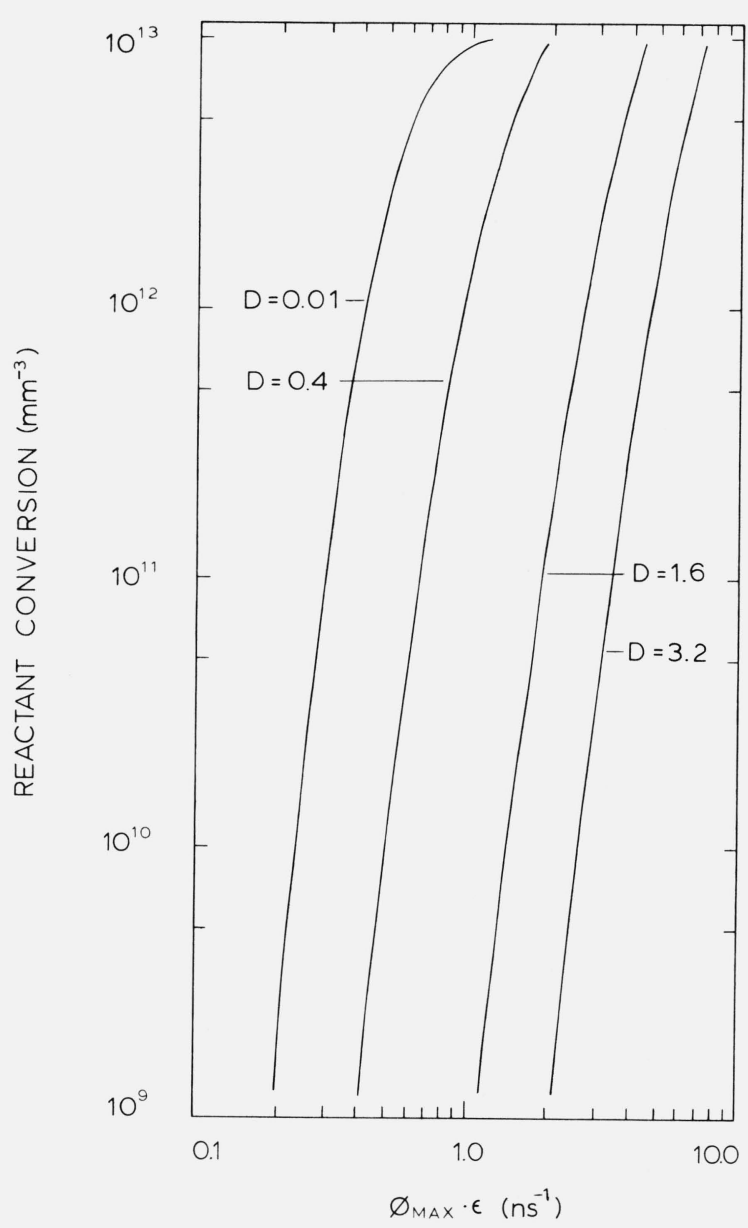

FiguRE 6. Plot of reactant to product conversion (molc $\left.\cdot \mathrm{mm}^{-3}\right)$ versus $\phi_{\max } \cdot \epsilon(n s)^{-1}$ (or intensity) as a function of varying amount of deactivation (parameter D varied)

Parameters $N$ and $s$ are 26 and 12 respectively.

\section{Model Versus Experiment}

The calculated product-intensity curve $N=26, S=12$ and $D=0.4$ (fig. 7), is plotted as the upper dashed curve in figure 8. It is positioned vertically so that the saturation point coincides with the experimentally determined value at about $3 \times 10^{-2}$ and is positioned horizontally so that it best fits the 


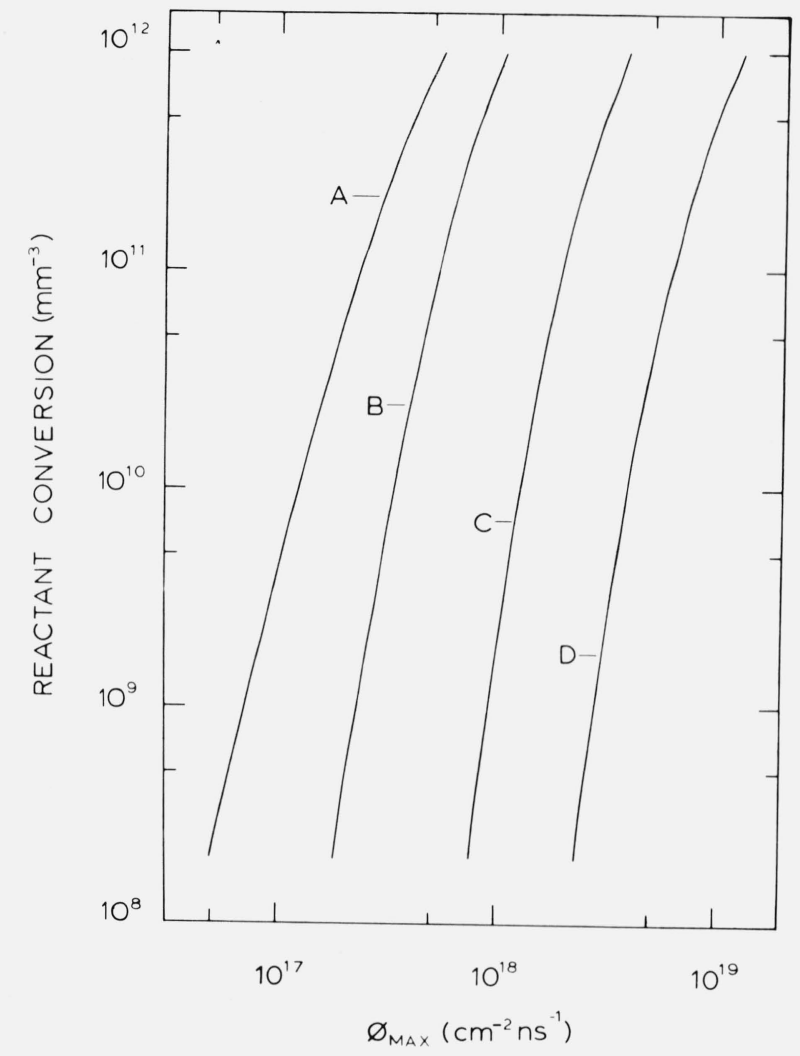

FIGURE 7. Computer simulation of reactant conversion versus $\phi_{\max } \cdot \boldsymbol{\epsilon}$. For all curves $D=0$ and $S=1$.

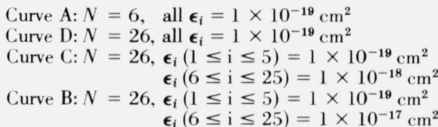

experimental points (taken from fig. 2). The apparent discrepancy at high conversions reveals the range where the photolysis volume is no longer constant. Here the yield tends toward a 1.5 power dependence arising from convolution by the conical geometry as discussed previously.

The overall agreement between the model calculation, upper dashed curve and experiment, solid circles, is seen to be only qualitatively satisfactory over the entire range below the saturation point.

The photometry experiments indicate that the astigmatic focal volume consists roughly of two regions of differing intensity. A small volume (of about $2 \%$ of the total volume) has an intensity approximately two-fold higher than the rest. At the lower intensities the effect of the intensity distribution is to sharply reduce the effective photolysis volume; at the lowest intensities the photolysis occurs mainly in the small "hot" spot. The measured yield-intensity points at the lowest intensity require a correction for the effect of the "hot" spot. This correction is attempted in figure 8 in the following way. The upper dashed curve is displaced vertically so that the saturation point is at about 2 percent of the total saturation volume and is displaced horizontally to the left corresponding to a factor of two in intensity. This lower curve approximately

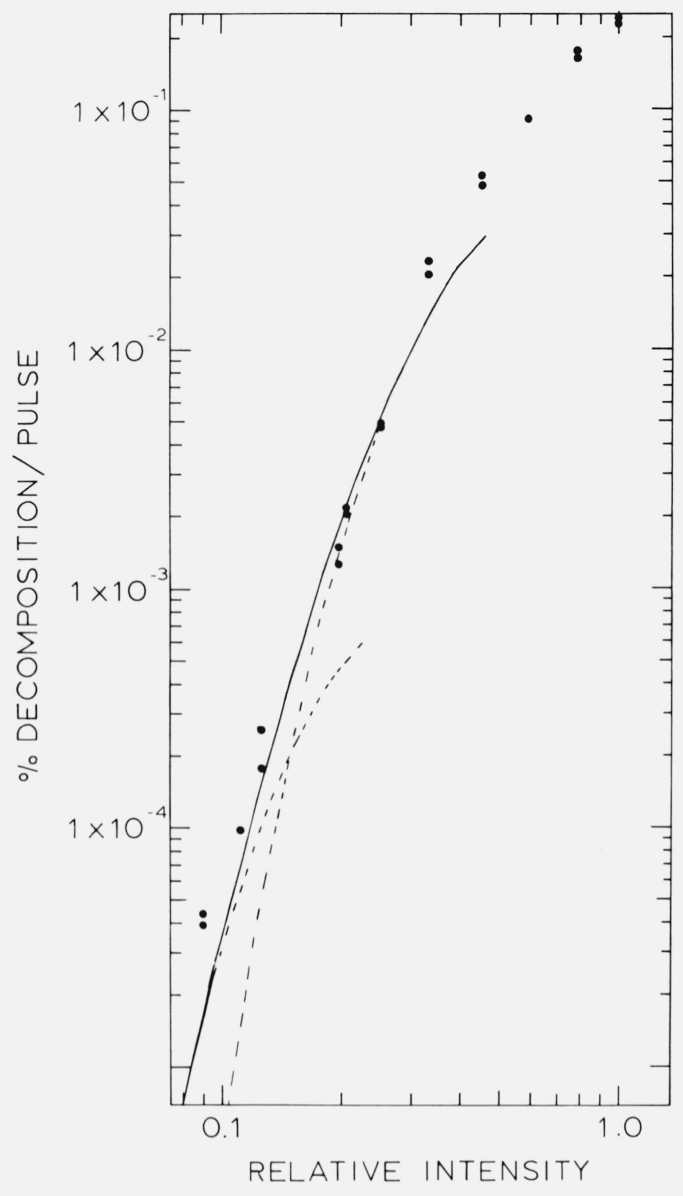

FigURE 8. Model versus experiment.

Solid points taken from figure 2. Upper and lower dashed curves: calculation for $N=26, S=$ 12 , and $D=0.4$. These curves are positioned as described in the text. Solid curve is the sum of the two dashed curves.

simulates the yield in the "hot" spot. The solid curve is the sum of the upper and lower dashed curves and represents the yield versus intensity dependence in this somewhat oversimplified representation of the experimental situation (two intensity photolysis zones).

The present model predicts that the product versus intensity dependence exhibits no threshold behavior. In contrast the results of Ambartzumian et al. [9] for dissociation of $\mathrm{SF}_{6}$ in the collision free regime clearly show threshold behavior, but more recent experiments performed in a collisional regime do not [21]. The present data, which are also taken in a collisional regime (figure 2), also do not reveal any apparent threshold behavior for photodissociation. Collisions must clearly play a dominant role in affecting the nature of the multiphoton dissociation process [32]. This is implied by the data of figure 9 which show that collisional processes significantly enhance the multiphoton dissociation process presumably by affecting the absorption and relaxation processes. At higher pressures deactivative processes dominate and the product yield falls off (as the present model predicts). We have not as yet attempted to treat the effect of pressure on the absorption process in the present model. 


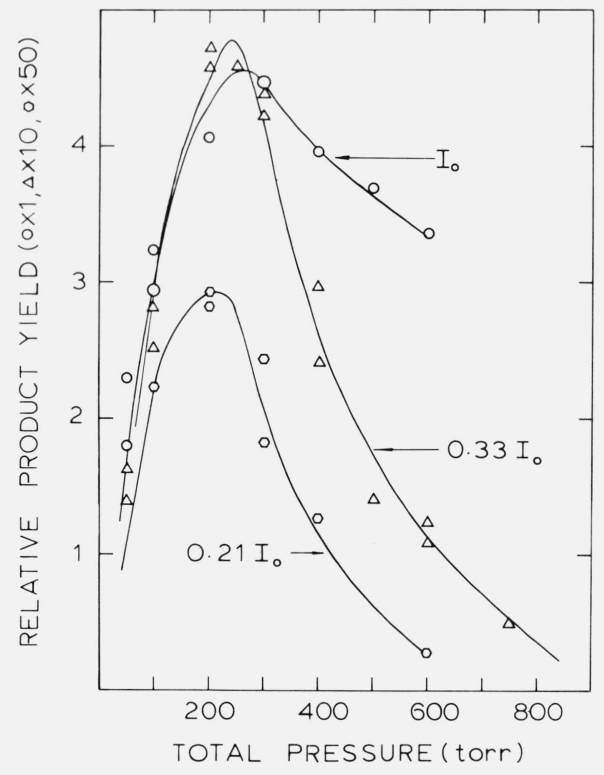

Figure 9. Plot of product yield versus total helium pressure at three intensities $\left(0.21 I_{0}, 0.33 I_{0}\right.$ and $\left.I_{0}\right)$

Experimental conditions: $\mathrm{P}_{\mathrm{C}_{2} \mathrm{H}_{4}}=0.625$ torr, photolysis at the $\mathrm{P} 14,10.6 \mu \mathrm{CO}_{2}$ laser line.

This yield-pressure behavior is not universal. Preliminary results on the photolysis of ethyl acetate, for example, show a yield-pressure relationship having a negative slope over the entire experimental range.

Figure 10 shows the dependence of the photodissociation yield as a function of wavelength in a collisional regime. The maximum yield is closely centered about the i.r. absorption band of $\mathrm{C}_{2} \mathrm{H}_{4}$. This result differs significantly from recent results obtained by Ambartzumian et al. [9], who found a distinct red shift for the photodissociation maximum relative to the absorption band of $\mathrm{SF}_{6}$ under collision free conditions.

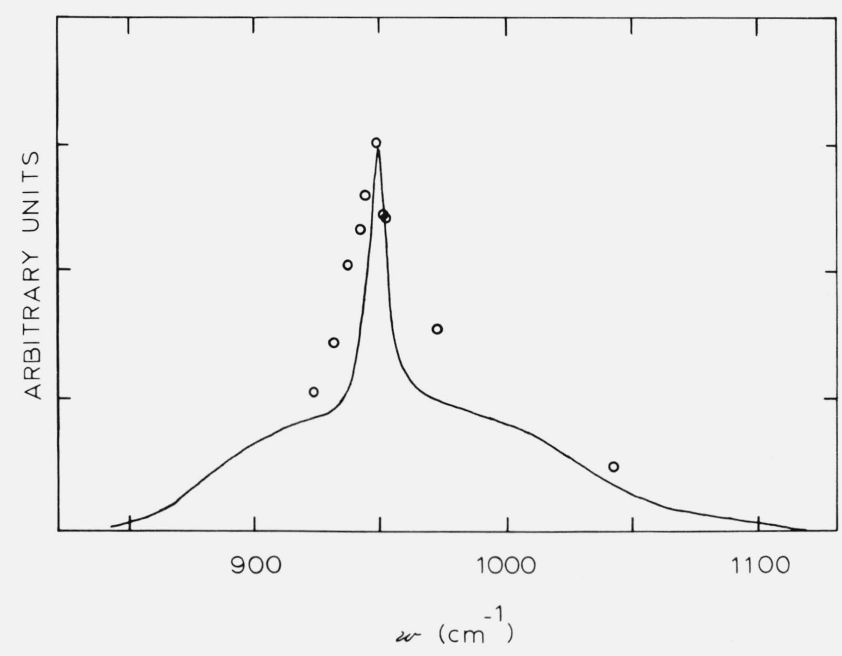

Figure 10. Points: Relative product yield versus laser line wavelength at approximately constant laser intensity.

Curve: extinction coefficient taken at low resolution, data from R. C. Golike, I. M. Mills, W. B. Person, and B. Crawford, Jr., J. Chem. Phys. 25, 1266 (1956).
A number of models describing the multiphoton dissociation process have been suggested. These are specifically designed to describe collision free multiphoton dissociation of molecules and may not be relevant to the present results. An early model of Bloembergen [8] assumes that coherent excitation processes are of paramount importance for pumping the first several vibrational levels. Levels higher than the first few were treated as a quasi-continuum because of the high density of states. Here it was assumed that rapid intramolecular relaxation occurs between states. Absorption processes occurring in the quasi-continuum were considered to be very efficient (molecules were assumed to be very efficiently excited from the quasi-continuum states into the dissociation continuum).

Recent models by Mukamel and Jortner [12, 14] and by Cotter [15] deal with more specific coupling mechanisms between energy states in the molecular manifold of states and coupling between these states and the radiation field. Resonant and/or near resonant transitions also are assumed (collision free regime). The multiphoton dissociation process should exhibit effects associated with the coherent nature of the driving laser pulse. Hougen has recently also proposed an interesting model based on a density matrix formulation which considers line broadening through collisions [10].

We employ a rate equation method in the present paper. Its application is advocated only to photolysis where there is strong intramolecular energy redistribution. The kinetic model contains only two molecular parameters, the phenomenological absorption coefficient, $\epsilon$, and the number of vibrational degrees of freedom in the molecule, $s$, which couple to the pumping radiation. Collisional deactivation is included in the model, but the yield-intensity curves are not strongly influenced by this process, except for the absolute yield at a particular laser intensity. We have not yet attempted to model the dependence of photochemical yield on the total pressure, which will require additional assumptions regarding the absorptivities. The parameters are adjusted in the present calculations, because the required absorption coefficients and detailed energy loss $(V-T)$ crosssections have not been measured. In principle these physical quantities are measurable.

The phenomenon of secondary photolysis occurring in the laser pulse, reported previously [16], can be treated by an extension of the present model. For example, the sequence

$$
\begin{gathered}
\mathrm{C}_{2} \mathrm{H}_{5} \mathrm{Cl} \stackrel{n h \nu}{\longrightarrow} \mathrm{C}_{2} \mathrm{H}_{4}+\mathrm{HCl} \\
\mathrm{C}_{2} \mathrm{H}_{4} \stackrel{m h \nu}{\longrightarrow} \mathrm{C}_{2} \mathrm{H}_{2}+\mathrm{H}_{2}
\end{gathered}
$$

occurs in a single laser pulse. Preliminary results demonstrate qualitative agreement between model and experiment for the $\mathrm{C}_{2} \mathrm{H}_{4} / \mathrm{C}_{2} \mathrm{H}_{2}$ product ratio as a function of laser intensity. Further work on this system is still in progress.

To further test the various models future experiments are necessary to probe:

(a) product yield dependence on the coherence of the laser pulse in a collisional as well as in a collision free regime.

(b) experiments in a collisional regime, covering a wider dynamic range of product yield versus intensity in order to further test for possible threshold behavior. 
(c) measurement of the average number of photons absorbed/molecule possibly through precise calorimetric measurements. In this way not only can the slopes of the product versus intensity dependence curves be used to test the model as done here but the position of the curves with respect to absolute $\left(\phi_{\max } \cdot \boldsymbol{\epsilon}\right)$ values are specified and this imposes an additional and final constraint on the predictions of the model.

(d) Time resolved measurements of product appearance during the laser pulse. The model implies such predictions but they are not given here because of the absence of any such measurements.

\section{References and Notes}

[1] Isenor, N. R., Merchant, V., Hallsworth, R. S., and Richardson, M. C., Can. J. Phys. 51, 1281 (1973).

[2] Ambartzumian, R. V., Letokhov, V. S., Ryabov, E. A., Chekalin, N. V., JETP Letters 20, 273 (1974).

[3] Ambartzumian, R. V., A. Gorokov, Yu., Letokhov, V. S., Makarov, G. N., ZhETF Pis. Red. (Sov.) 21, 375 (1975).

[4] Following references [4-15] are only a representative sampling of all of the papers that have appeared. Kudrin, L. P., and Mikhailova, Yu. V., Sov. Phys.-JETP 41, 1049 (1976).

[5] Bloembergen, N., Optics Comm. 15, 416, (1975).

[6] Akulin, V. M., Alimpiev, S. S., Karlov, N. V., and Shelepin, L. A., Sov. Phys.-JETP 42, 427 (1976).

[7] Letokhov, V. S., and Makarov, A. A., Optics Comm. 17, 250 (1976).

[8] Larsen, D. M., and Bloembergen, N., Optics Comm. 17, 254 (1976).

[9] Ambartzumyan, R. V., Gorokhov, Yu. A., Letokhov, V. S., Makarov, G. N., and Puretskii, A. A., JETP Letters 23, 22 (1976).

[10] Hougen, J. T., J. Chem. Phys. 65, 1035 (1976).

[11] Faisal, F. H. M., Optics Comm. 17, 247 (1976)

[12] Mukamel, S., and Jortner, J., Chem. Phys. Lett. 40, 150 (1976).

[13] Stone, J., Goodman, M. F., and Down, D. A., Chem. Phys. Lett. 44, 411 (1976).

[14] Mukamel, S., and Jortner, J., J. Chem. Phys. 65, 5204 (1976).

[15] Cotter, T. P., J. Chem. Phys. (in press).

[16] Braun, W., and Tsang, W., Chem. Phys. Lett. 44, 354 (1976).

[17] Ledford, A. E., and Braun, W., Rev. Sci. Instrum. 48, 537 (1977).

[18] Born, M., and Wolf, E., Principles of Optics, 3rd ed., (Pergammon Press, Oxford, 1965).

[19] Bell, R. J., and Romero, H. V., Appl. Optics 9, 2341 (1970).

[20] Marquet, L. C., Appl. Optics 10, 960 (1971).

[21] Campbell, J. D., Hancock, G., and Welge, K. H., Chem. Phys. Lett. 43, 581 (1976).
[22] Speiser, S., and Jortner, J., Chem. Phys. Lett. 44, 399 (1976).

[23] Braun, W., Bass, A. M., and Pilling, M., J. Chem. Phys. 52, 5131 (1970).

[24] Rowland, F. S., Lee, P. S., Montague, D. C., and Russell, R. L., Disc. Faraday Soc. 53, 111 (1972).

[25] Gutman, D., Braun, W., and Tsang, W., J. Chem. Phys. 67, 4291 (1977).

[26] Robinson, P. J., and Holbrook, K. A., "Unimolecular Reactions," (Wiley-Interscience, London, 1972).

[27] This is not a unique way of defining an average absorption coefficient. Alternatively we could have defined the average absorption coefficient as the sum of microscopic cross-sections from each sublevel of the $i$ th energy index to each sublevel of the $(i+1)$ energy index divided by the number of sublevels of the $(i+1)$ level. This is equivalent dividing one then the other of the bracketed terms in equation (I) by $B_{i}$ and $B_{i+1}$ respectively, e.g.,

$$
\left[Z_{i-1}-B_{i} Z_{i}\right] \text { becomes }\left[Z_{i-1} B_{i}^{-1}-Z_{i}\right]
$$

Defining the average absorption coefficient this way leads to quantitatively similar results as the expression in the text.

[28] The units of $\phi_{\max }$ are photons/nanosecond $-\mathrm{cm}^{2}$. Calculations were made taking $2 \tau$ the pulse width to be 100 nanoseconds. The number of photons $\mathrm{cm}^{-2}$ incident during the laser pulse $\phi$ (total) can be calculated by integrating $\phi(t)$ over the pulse duration, $2 \tau$, and is related to $\phi(\max )$ by $\phi($ total $)$ photons $/ \mathrm{cm}^{2}=62.5 \times \phi(\max )$

[29] Glick, H. S., Symp. Combust., 7th London, Oxford 1958, 76, (1959)

[30] The parameter $s$, in a unimolecular theory context defines the lifetime of a molecule with respect to energy in excess of the dissociation (activation) energy. In the present model, since the molecule is assumed to dissociate irreversibly at some energy level $i=N$, the significance to the parameter $s$ pertains only to the absorption and stimulated emission process. That is to say that for a "real" molecule one would have to increase $N$ as one increases s to simulate the correct situation.

[31] For comparison purposes our laser delivers 0.5 joules per pulse focused into an area of $5 \times 10^{-4} \mathrm{~cm}^{2}$, therefore it delivers $5 \times 10^{22}$ photons $\mathrm{cm}^{-2}$ per pulse. Our program is written so that

$$
\text { Photons }(\text { Total }) \# / \mathrm{cm}^{2}=66.6 \times \phi(\max )
$$

Therefore $\phi(\max )=7.5 \times 10^{20}$ photons $/ \mathrm{cm}^{2} \cdot$ nanoseconds

From one of our curves, $N=26, S=6, D \approx 0, \phi(\max \cdot \epsilon \approx 1.0)$ (near saturation), therefore $\epsilon_{i}$ is $\sim 1.3 \times 10^{-21} \mathrm{~cm}^{2}$. A moderate absorption coefficient is about $3 \mathrm{~atm}^{-1} \cdot \mathrm{cm}^{-1}$ or 1.22 $\times 10^{-19} \mathrm{~cm}^{2}$, just about two orders of magnitude larger than the $\epsilon$ assumed. This appears to be a reasonable number for an absorption coefficient in the "tail" of a Lorentzian profile.

[32] Patel, C. K. N., and Slusher, R. E., Phys. Rev. Lett. 19, 1019 (1967). 